\title{
VHDL Design for Image Segmentation using Gabor filter for Disease Detection
}

\author{
Rucha R. Thakur ${ }^{1}$, Swati R. Dixit ${ }^{2}$ and Dr.A.Y.Deshmukh ${ }^{3}$ \\ ${ }^{1}$ Department of Electronics \& Telecommunication Engineering, PG Student, \\ G.H.Raisoni College of Engineering, Nagpur, India \\ rucha. thakur25@yahoo.com \\ ${ }^{2}$ Department of Electronics \& Telecommunication Engineering, Research Scholar, \\ G.H.Raisoni College of Engineering, Nagpur, India \\ srdixit2008@yahoo.com \\ ${ }^{3}$ Department of Electronics Engineering, Professor, G.H.Raisoni College of Engineering, \\ Nagpur, India \\ amolydeshmukh@yahoo. com
}

\begin{abstract}
Tonsillitis, Tumor and many more skin diseases can be detected in its early-state and can be cured. For this a new idea for efficient Gabor filter design with improved data transfer rate, efficient noise reduction, less power consumption and reduced memory usage is proposed in this paper. The filter design is suitable for detecting the early stages of disease using textural properties of anatomical structures. The code for Gabor filter will be developed in VHDL using Modelsim and then implemented on SPARTAN-3E FPGA kit. These systems must provide both highly accurate and extremely fast processing of large amounts of image data.
\end{abstract}

\section{Keywords}

Segmentation, Medical image, Gabor algorithm, CORDIC algorithm, FPGA

\section{INTRODUCTION}

Image segmentation [1] is the process of partitioning a digital image into multiple segments i.e. sets of pixels. Segmentation of images by using textural property of anatomical structures and regions of interest has a crucial role in most medical imaging applications. The segmented image is more meaningful and easier to analyze.

For medical images, Color image [2] segmentation [3] and cell counting system is preferred because the gray levels alone may not be sufficient to perform accurate medical image segmentation, as many soft tissues have overlapping gray level ranges. Thus the use of the textural properties of the anatomical structures could be useful.

For this purpose a customized 2D Gabor Filter for RGB color image [6] segmentation will be designed. It has proved to be an effective segmentation tool with improved data transfer rate, efficient noise reduction, less power consumption and reduced memory usage .Gabor function [7]

DOI : $10.5121 /$ vlsic.2012.3218 
International Journal of VLSI design \& Communication Systems (VLSICS) Vol.3, No.2, April 2012

locates the texture features in the spatial domain. Gabor filters have proved to be an effective segmentation tool because of two major factors as: Their capability to achieve optimal uncertainty in both space and frequency, and their similarity with primary visual cortex of mammals.

We focus on detecting main features of disease and create a resulting image showing affected area on MATLAB. The Gabor Filter for color image segmentation will be coded using VHDL in Modelsim and will be implemented in SPARTAN-3E FPGA. Field Programmable Gate Array (FPGA) [8] technology [12] has become a viable target for the implementation of algorithms suited to image processing applications. Finally the segmented image will be observed on MATLAB.

\section{DeSign MethodologY}

The design approach will be divided in six modules as described below

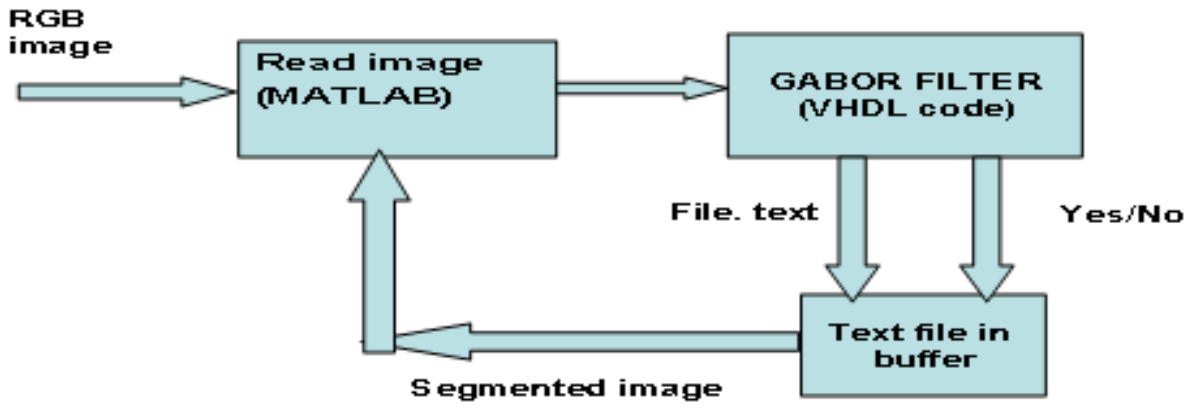

Figure (1): Block Diagram of proposed plan

\subsection{MATLAB IMAGE READING MODULE}

This is a simple image reading and resizing module written in MATLAB. It reads two images from database for comparison. One of which is healthy image (figure (2)) and another having disease features (figure (3)). The comparison will generate a test input file which we can use as input to VHDL module.

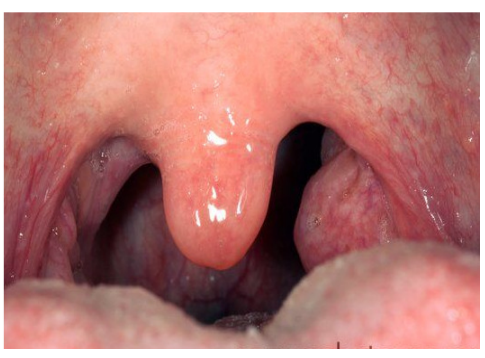

Figure (2): Healthy Tonsils

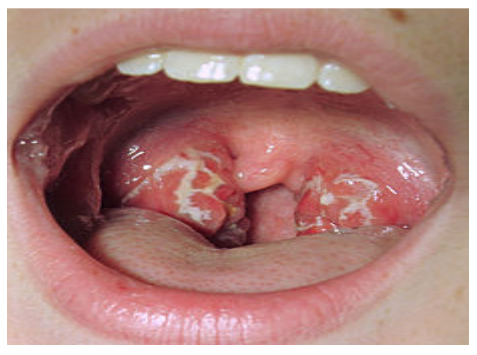

Figure (3): Disease affected tonsils 
International Journal of VLSI design \& Communication Systems (VLSICS) Vol.3, No.2, April 2012

\subsection{GABOR AlgORITHM}

Our Gabor-type filter designed with Gabor algorithm is used as the processing unit in a disease detection module. Gabor Filters have received considerable attention because the characteristics of certain cells in the visual cortex of some mammals can be approximated by these filters [14].

Gabor filters are a large set of linear filters, having the impulse response defined as a harmonic function multiplied by a Gaussian function with various orientations. It can be viewed as a sinusoidal plane of particular frequency and orientation, modulated by a Gaussian envelope. The space domain representation of the complex 2D Gabor filters [15] (or functions) is given by

$$
\mathrm{h}(\mathrm{x}, \mathrm{y})=\mathrm{s}(\mathrm{x}, \mathrm{y}) \mathrm{g}(\mathrm{x}, \mathrm{y})
$$

Where $\mathrm{s}(\mathrm{x}, \mathrm{y})$ is a complex sinusoid, known as a carrier and $\mathrm{g}(\mathrm{x}, \mathrm{y})$ is a 2-D Gaussian shaped function, known as envelope and it's spatial extent is given by the parameter $\sigma g$. These are defined as follows,

$$
\begin{aligned}
& \mathrm{s}(\mathrm{x}, \mathrm{y})=e^{\mathrm{j}\left(\mathrm{w}_{\mathrm{x}} \mathrm{x}+\mathrm{w}_{\mathrm{y}} \mathrm{y}\right)} \\
& \mathrm{g}(\mathrm{x}, \mathrm{y})=\frac{1}{2 \pi \sigma^{2}} e^{-\frac{\mathrm{x} 2+\mathrm{y} 2}{2 \pi \sigma^{2}}}
\end{aligned}
$$

Thus the 2-D Gabor filter equation can be written as:

$$
\mathrm{h}(\mathrm{x}, \mathrm{y})=\frac{1}{2 \pi \sigma^{2}} e^{-\frac{\mathrm{x} 2+\mathrm{y} 2}{2 \pi \sigma^{2}}} e^{\mathrm{j}\left(\mathrm{w}_{\mathrm{x}} \mathrm{x}+\mathrm{w}_{\mathrm{y}} \mathrm{y}\right)}
$$

The equivalent frequency representation of Gabor filters is:

$$
\mathrm{h}(\mathrm{u}, \mathrm{v})=\exp \left\{-2 \pi^{2} \sigma \mathrm{g}^{2}\left[(\mathrm{u}-\mathrm{U})^{2}+(\mathrm{v}-\mathrm{V})^{2}\right]\right\}
$$

The Gabor filter is essentially a bandpass filter centered at $(\mathrm{U}, \mathrm{V})$ in the frequency domain, with bandwidth determined by $\sigma g$. Its radial center frequency measured in cycles/ image, $\mathrm{fc}=$ $\sqrt{ } \mathrm{U} 2+\mathrm{V} 2$, is oriented (in radians) from the u-axis with $\theta=\tan -1(\mathrm{~V} / \mathrm{U})$. We assume, for the simplicity, that the Gaussian $g(x, y)$ is a symmetrical function.

The Gabor filter bank is obtained by generating Gabor filters for all directions from 0 to $360^{\circ}$ and varying the frequency with the fc factor. The representation of the generated filters in the frequency domain is shown in Figure (4). 


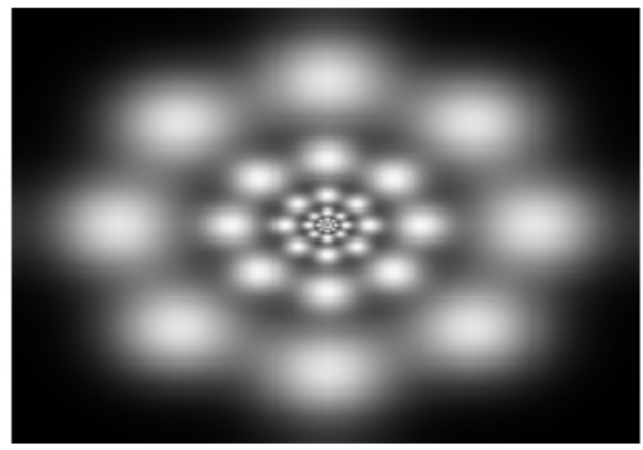

Figure (4): The frequency domain of Gabor filter bank

This Gabor algorithm [15] in equation (2) discussed above, will be use to implement a Gabor Filter in VHDL. This is basically implementation of certain equations of the Gabor filter, shown above, to provide an output (image segmentation) which gives better results as compare to general image segmentation.

\subsubsection{CORDIC Algorithm}

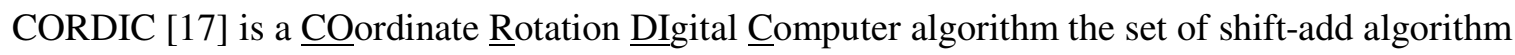
collectively known as CORDIC algorithm for computing a wide range of functions including trigonometric, hyperbolic, logarithmic and linear functions. It is introduced in 1959 by Jack E. Volder.

As we observe the Gabor Filter equation, the implementation is quite complex as the complex exponential term is present there. This term is divided in two kernels. Even kernel is cosine modulated and the odd kernel is sine modulated as $e^{(j \theta)}=\cos \theta+\mathrm{j} \sin \theta$ and hence two filers are 90 degrees out of phase. These trigonometric functions which are based on vector rotations are implemented using Iterative shift \& add operation. No Multiplication is required and hence Delay/Hardware cost is reduced comparable to division or square rooting. It is a Hardware Efficient Algorithm. The data format consists of two 16-bit words which are used for the even and odd kernels of Gabor filter equation. The 16-bit words consist of a 4-bit integer part and 12bit fractional part. The image pixels are represented by an 8-bit number which is stored in lookup table in do file format. This Gabor filter is designed with CORDIC algorithm reduces the time required for computation making the system fast.

\subsection{IMAGE SEGMENTATION MODULE}

This would apply the Gabor filter to the input image, and provide a segmented output in the form of a 2D array. 
International Journal of VLSI design \& Communication Systems (VLSICS) Vol.3, No.2, April 2012

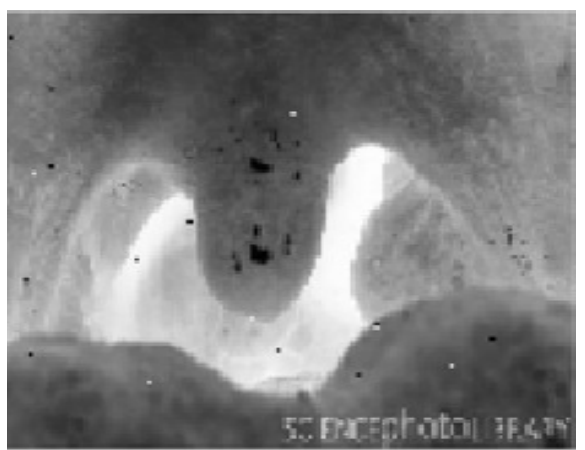

Figure (5): Segmented Healthy Tonsils

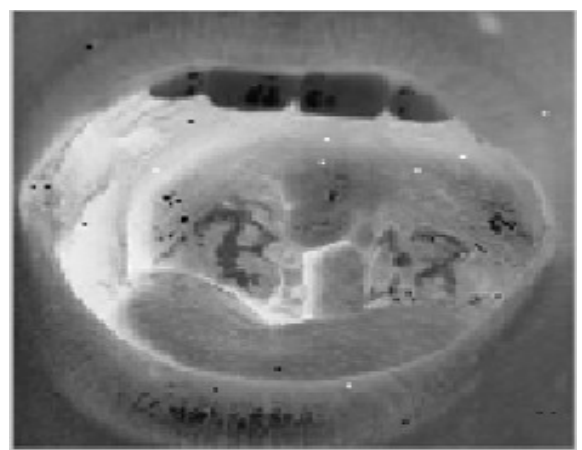

Figure (6): Segmented Disease affected tonsils

\subsection{DiSEASE DETECTION MODULE}

Depending upon the output of image segmentation module i.e. (a) the value in matrix showing total size of disease affected area and (b) color of disease, this module will detect if disease (Tonsillitis) is present in the input image or not, and provide an output depicting the same highlighting only tonsil area. The main concept used in this is to extract only the overlapping area in given two images helps in highlighting the disease area and gives the result in percentage that how much percent the disease is present. With this we can also able to identify the stage of disease by observing the intensity value of pixel in each segment.

\subsection{VHDL OUTPUT MODULE}

The output from step 4 would be stored in a text file and an output file would be generated (figure (7)). This would contain the actual output image.

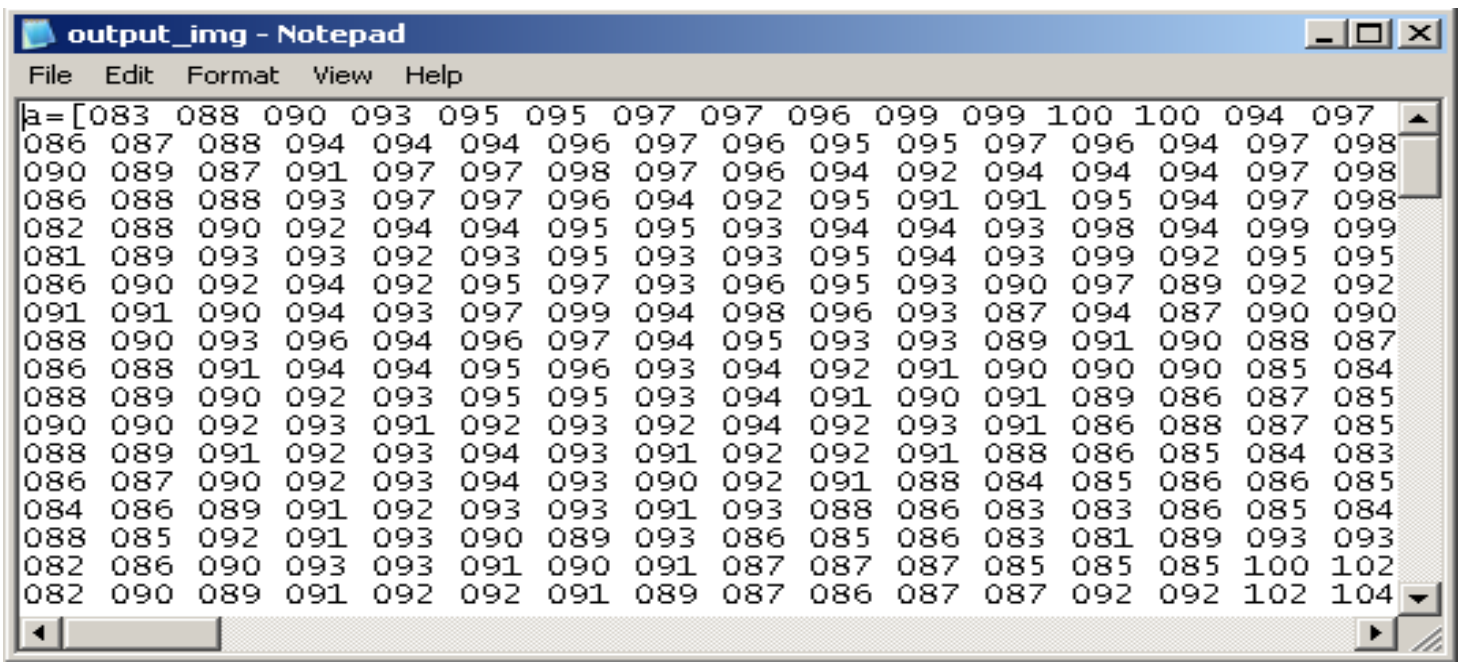

Figure (7): Text file showing output 
International Journal of VLSI design \& Communication Systems (VLSICS) Vol.3, No.2, April 2012

\subsection{FINAL RESULT DISPLAY MODULE}

This would be a simple MATLAB code named as post file.m which would take the difference output from step 5 and display it on the MATLAB screen in the form of a percentage. Here it is showing $68.36 \%$ tonsillitis is detected as it is greater than $60 \%$ which is the limit of healthy tonsils.

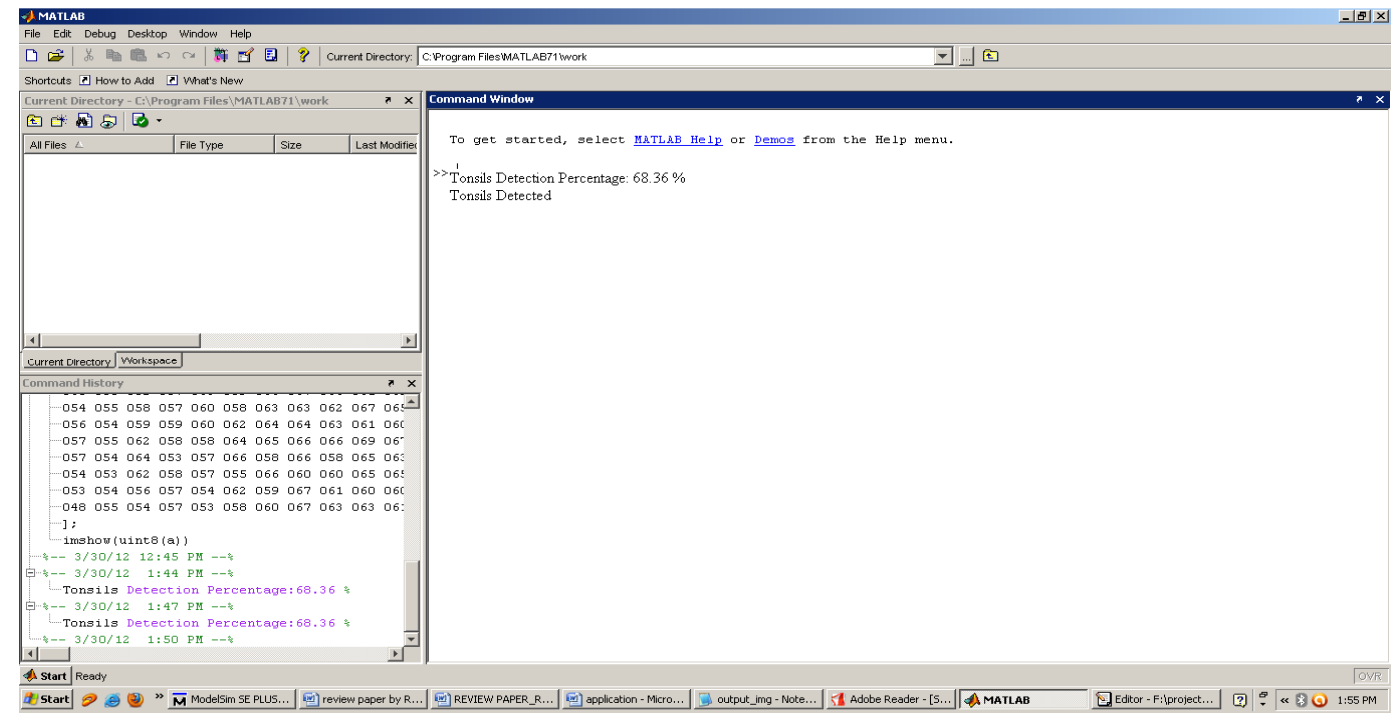

Figure (8): MATLAB showing final result

\section{FLOW OF DESIGN}

The overall design is revised in the flowchart shown below in figure (9). It shows different Modules designed in MATLAB (modules outside hashed lines) and VHDL (modules inside hashed lines).

The image is captured using USB image sensor [8], where it is resized. Next the Gabor filter is applied to the image for segmentation. This Gabor filter is designed with CORDIC algorithm for computation \& making the system fast.

The segmented image can be observed on image display module within microseconds. The disease detection module, which is designed in VHDL, compares the two images and gives intensity values of disease affected area. This will be helpful in identifying the stage of disease finding the percentage of disease affected area.

\section{ANALYSIS AND DEVELOPMENT}

In developing algorithm some considerations must be taken, such as: properties and constraints. In software base, these properties are: performance (accuracy and speed), complexity, size of code, size of templates, difficulty of development, dependency, and in hardware base these properties are: performance, size of block/modules, and size of templates. And in implementing 
International Journal of VLSI design \& Communication Systems (VLSICS) Vol.3, No.2, April 2012

software algorithm [8] into hardware base some constraints must be taking care, such as: memory, component/block device, module dependency, difficulty of development, interfacing and handshaking, licensing, etc.

Initially the work will be simulated using VHDL and then implemented on SPARTAN-3E FPGA.

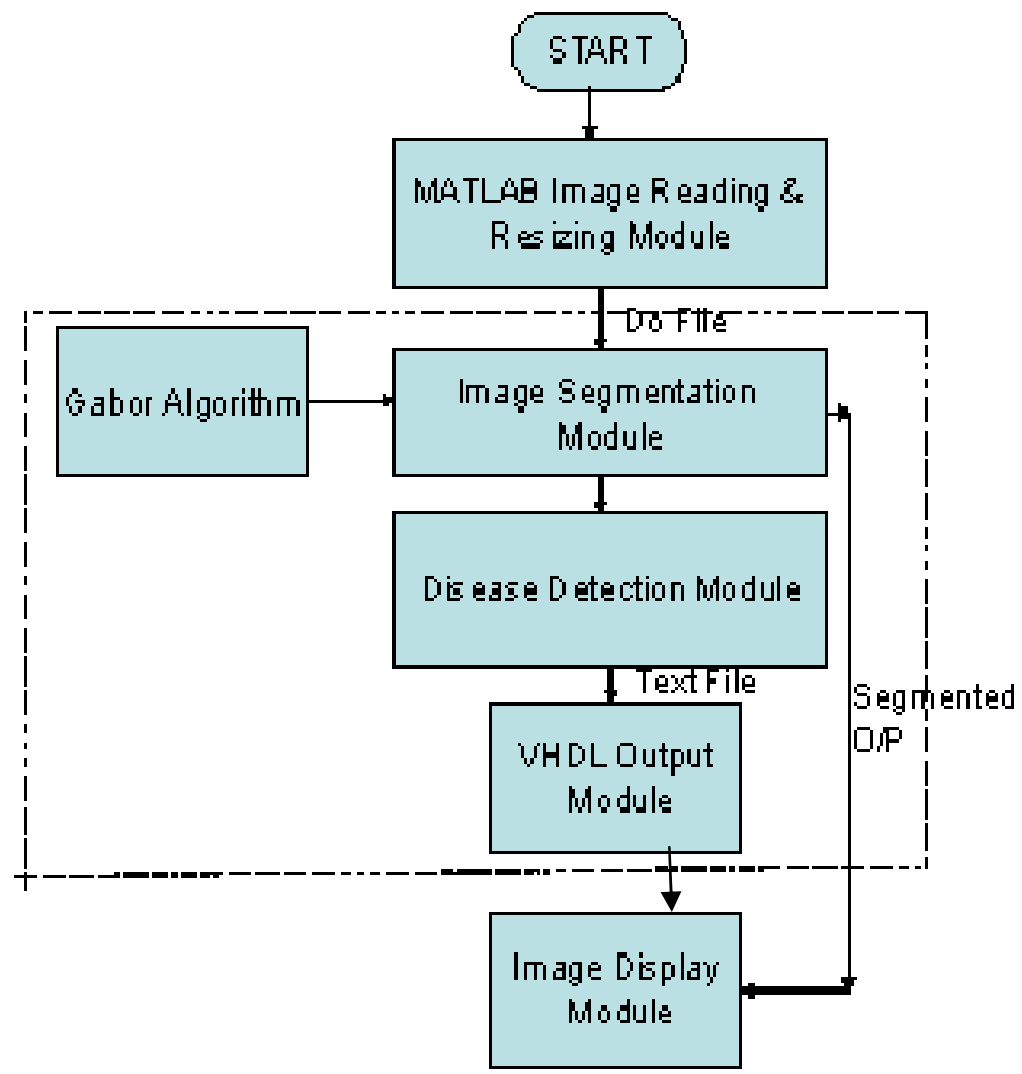

Figure (9): Data flow of overall module

\section{RESULTS AND DISCUSSION}

The input images used here are the tonsils images. Instead of this RGB image of tonsillitis we can use any RGB image of skin disease as it is generalized algorithm with some changes. The image size is fixed to $128 \times 128$. Thus each input image will be resized and converted to grayscale image (figure (5) (6)). This module generates $128 \times 128 \times 8$ number of lines showing the pixel values in V-sim format suitable to use in Modelsim i.e. to interface VHDL code with MATLAB code. Three Gabor filter for R, G \& B are designed to filter each component in image to give the noise free result. After obtaining the segmented image as output using Gabor filter we found that noise contents are reduced to great extent locating the exact region of tonsil area. The pixel values of the input image are obtained and provided serially to disease detection module. Here the comparison is performed between two samples giving the desired result in percentage as explained above. This design further can be implemented on VERTEX / SPARTAN-3E FPGA kit [8]. 
International Journal of VLSI design \& Communication Systems (VLSICS) Vol.3, No.2, April 2012

\section{CONCLUSIONS}

In this paper, a concept of VLSI architecture for skin related disease detection is proposed. It will improve data transfer rates, provide efficient noise reduction, less power consumption and require less memory storage. The Gabor filter is an efficient tool to get all requirements as mentioned above. The processing time required for simulation is very less as compared to software simulation because of the use of CORDIC algorithm, thus offers much greater speed than a software implementation. This concept will be helpful in detecting early stage of disease and saving the lives of peoples.

\section{REFERENCES}

[1] T.Ratha Jeyalakshmi, K.Ramar, "Segmentation of Uterine Fibroid Using Morphology: An Automatic Approach,” International conference on Intelligent Agent \& Multi-Agent Systems, July 2009

[2] "Color image processing," by S. J. Sangwine, Electronics \& communication engineering journal October 2000

[3] Myung-Eun Lee', Soo-Hyung Kim', Sun-Worl Kim2 and Sung-Ryul Ohl," Automatic Segmentation Methods for Various CT Images Using Morphology Operation and Statistical Technique," IEEE $3^{\text {rd }}$ International conference on Intelligent computer communication and processing (ICCP), 2007

[4] Jia Xin-Wang, Ting Ting-Zhang," CT Image Segmentation by using a FHNN Algorithm Based on Genetic Approach,” International conference on Bioinformatics and Biomedical Engineering, pp.1-4, July 2009.

[5] Thomas P.Weldon and William E. Huggins, "Designing Multiple Gabor Filters for Multi-Texture Image Segmentation,” Optical Engineering, Vol. 38 No. 9, pp. 1478-1489, 1999.

[6] Pranithan Phensadsaeng, Werapon Chiracharit and Kosin Chamnongthai, "A VLSI Architecture of Color Model-based Tonsillitis Detection'," 2009 IEEE

[7] Malarkhodi.S, Dr.R.S.D.Wahida Banu, Malarvizhi.M, "VLSI Implementation of Uterus Image Segmentation Using Multi-Feature EM Algorithm Based on Gabor Filter'," 2010 Second International conference on Computing, Communication and Networking Technologies

[8] Lingga Henan, Sunny AriefSudiro, Eri Prasetyo Wibowo "Hardware Implementation of Fingerprint Image Thinning Algorithm in FPGA Device," 2010 International Conference on Networking and Information Technology

[9] Rafael C. Gonzalez, Richard E. Woods, "Digital Image Processing", Third Edition, University of Tennessee, pp., $443-445,598-605,1992$.

[10] Anil K. Jain, "Fundamentals of Digital Image Processing", University of California, pp. 1 - 7, 244 252, $347-374,1997$.

[11] Arthur R. Weeks, "Fundamentals of Electronic Image Processing", University of Central Florida, pp. $426-427,2005$.

[12] Anthony Edward Nelson, "Implementation of Image Processing Algorithms on FPGA Hardware" ,Master of Science in Electrical Engineering, May 2000. 
International Journal of VLSI design \& Communication Systems (VLSICS) Vol.3, No.2, April 2012

[13] Rosas R.L., de Luca A., Santillan F.B., "SIMD architecture for image segmentation using Sobel operators implemented in FPGA technology," International Conference on Electronics and Electrical Engineering.2005, Sept. 2005, pp.77-80

[14] Thomas P. Weldon and William E. Huggins, "Designing Multiple Gabor Filters for Multi-Texture Image Segmentation,” Optical Engineering, Vol. 38 No. 9, pp. 1478-1489, 1999.

[15] "Implementation of Gabor-type Filters on Field Programmable Gate Arrays" by Ocean Y. H. Cheung1, Philip H. W. Leong1, Eric K. C. Tsang2 and Bertram E. Shi2, 1The Chinese University of Hong Kong, Shatin, New Territory, Hong Kong, 2University of Science and Technology, Clear Water Bay, Kowloon.

[16] "A survey of CORDIC algorithms for FPGA based systems" by Ray Andraka, North Kingston. 